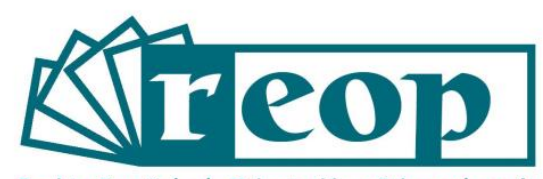

Revista Española de Orientación y Psicopedagogía

\title{
PERCEPCIÓN SOCIAL DE LA ORIENTACIÓN ESCOLAR EN ORIENTADORES DE BOGOTÁ 1
}

\section{SOCIAL PERCEPTION OF THE SCHOOL GUIDANCE IN BOGOTÁ'S SCHOOL COUNSELORS}

\author{
Oscar Gilberto Hernández Salamanca² \\ Departamento de Psicopedagogía, Universidad Pedagógica Nacional de Colombia. Bogotá, \\ Colombia
}

\section{RESUMEN}

Se presentan los resultados de una encuesta de percepción social sobre la orientación escolar diligenciada por 1007 orientadores de Bogotá. Esta encuesta se realizó con el apoyo de la Secretaría de Educación de la ciudad en el marco del Congreso de Orientación y Asesoría Escolar realizado en 2018. Estuvo compuesta por 10 ítems con respuesta tipo Likert sobre aspectos relativos a la actividad

1 Fuente de financiación: Artículo de investigación derivado del programa de estudio sobre actitudes hacia la orientación educativa en Colombia. Eje de Orientación Educativa del Departamento de Psicopedagogía de la Universidad Pedagógica Nacional. Se agradece a Angy Milena Caicedo, profesional de la Secretaría de Educación Distrital de Bogotá, quien facilitó el diligenciamiento de las encuestas por medios virtuales.

2 Correspondencia: Oscar Gilberto Hernández Salamanca. Magíster en Ciencias Sociales con Mención en Educación. (FLACSO). Departamento de Psicopedagogía. Universidad Pedagógica Nacional de Colombia. Calle 72 \#11-86. Bogotá, Colombia. Oficina 223. Tel: (57-1) 5941894 ext. 306 - 320. Email: oghernandezs@pedagogica.edu.co 
profesional. Se compararon las respuestas según la formación de pregrado del orientador, ya que en Colombia ese cargo está abierto a distintas profesiones. Los datos se analizaron en términos de variables nominales y ordinales dada la naturaleza de las escalas Likert y con el estadístico chi cuadrado. Entre los resultados se destacan: (a) La percepción social general de los orientadores hacia la orientación es favorable pese a las dificultades que enfrentan en su trabajo. Dicha favorabilidad es consecuencia de la reflexión sobre su práctica. (b) La formación de pregrado del orientador influye en la percepción de la orientación como una actividad docente X2 (12, N=1007) $=32.152, \mathrm{p}<0.05$. Aunque no todos los orientadores participantes se ven a sí mismos como maestros, en sus actividades promueven procesos de formación. Esto coincide con el panorama de la investigación correspondiente y fundamenta discusiones frente a la relevancia de la formación in situ de los orientadores y a la dimensión pedagógica de su práctica.

Palabras clave: Orientación escolar, percepción social, psicopedagogía, asesoramiento.

\begin{abstract}
This paper presents the results of a survey of social perception on school guidance completed by 1007 Bogotá's counselors. This survey was carried out with the support of the Ministry of Education of the city within the framework of the School Counseling and Advisory Congress held in 2017. It was composed of 10 items with a Likert-type response on aspects related to professional activity. The answers were compared according to the undergraduate training of the counselor, since in Colombia that position is open to different professions. The data were analyzed in terms of nominal and ordinal variables given the nature of the Likert scales and the chi-square statistic. Among the results are: (a) the general social perception of counselors towards guidance is favorable despite the difficulties they face in their work. Said favorability is a consequence of the reflection on its practice. (b) The undergraduate training of the counselor influences the perception of the orientation as a teaching activity $\mathrm{X} 2(12, \mathrm{~N}=1007)=32,152, \mathrm{p}<0.05$. Although not all participating counselors see themselves as teachers, in their activities promotes training processes this coincides with the panorama of the corresponding research and bases discussions on the relevance of the in-situ training of counselors and the pedagogical dimension of their practice.
\end{abstract}

Key Words: Educational guidance, social perception, psychopedagogy, counselling.

\title{
Introducción
}

La orientación escolar en Colombia es un campo muy heterogéneo. Aunque su origen está ligado a la formación para el trabajo, reflejado en la orientación vocacional, con el tiempo fue adquiriendo varios matices. En la actualidad coexisten en su práctica distintas profesiones, en las que prevalecen la psicología, la licenciatura en psicopedagogía y el trabajo social. Asimismo en ella confluyen distintas 
teorías y saberes que se entremezclan de múltiples maneras, originando un caleidoscopio difícil de caracterizar. La investigación académica en este campo recientemente comienza a impulsarse en el país.

La legislación vigente sobre la actividad de los orientadores muestra una distancia entre sus funciones legales y su trabajo en escuelas y colegios (Amadeo, 2007; Martínez, 2002). Pese a que el Ministerio de Educación Nacional y las entidades territoriales han reglamentado sus funciones, en la vida cotidiana de las instituciones los orientadores realizan actividades extremadamente diversas. Estas se derivan de una disputa simbólica entre la formación de pregrado del orientador y las expectativas de los demás miembros de las comunidades escolares, principalmente de los rectores (Hernández, 2016).

La orientación escolar es una actividad que está profundamente ligada al contexto en el que se desarrolla. Bejarano y Huertas (2017) argumentan que esto se debe a que la orientación escolar de Colombia tiene un perfil dirigido a fomentar actividades de corte sociocultural para atender problemas socialmente relevantes. De allí que a los departamentos de orientación de las escuelas colombianas se trasladen las grandes preocupaciones sociales de cada época: vocación, educación sexual, convivencia, y más recientemente, la construcción de la paz.

La singularidad del contexto puede investigarse de modo paradójico, rastreando algunos posibles elementos comunes. Esto sentaría una base para comprender más estructuralmente lo que significa la orientación escolar en contexto. Es importante contar con ese tipo de elementos para, eventualmente, organizar programas de investigación pertinentes en este campo. Por ejemplo, aún se desconoce con detalle el modo en que se valora al orientador en la escuela, o su relación con los demás maestros, o lo que piensan sobre las políticas educativas que los afectan.

Una manera de hacerlo es estudiando la percepción social de los mismos orientadores hacia su área de trabajo. Describir las ideas implícitas de la orientación escolar desde la perspectiva de los orientadores es sustancial, entre otras razones, porque son ellos quienes enfrentan las vicisitudes propias de esa actividad en los colegios del país. Igualmente esta información establecería rutas de investigación en los centros universitarios dedicados a este problema.

La percepción social es el conjunto de juicios elementales que se elaboran sobre las características de personas o fenómenos. En ellos están implícitos los significados e interpretaciones que de estos se construyen a través de la experiencia, y que se materializan en creencias, valoraciones y emociones (Nater y Zell, 2015; Myers, 2000). Una parte importante del comportamiento humano está ligado a este hecho porque dependiendo del modo en que se percibe el ambiente social y a las demás personas se privilegiarán unas acciones sobre otras.

Los estudios sobre la percepción social de la orientación escolar en el contexto latinoamericano muestran una favorabilidad mesurada (Campo y Labarca, 2009; Cano, Mayoral, Liesa y Castellano, 2013; Castillo y Torres, 2013; Hincapié y Arbeláez, 1979; Rodríguez, 2014). Mesurada porque si bien se reconocen los aspectos positivos de la orientación escolar también se registra la ambigüedad de sus propósitos, funciones, teorías y prácticas. En general se percibe favorablemente la orientación escolar aunque no se conocen con detalles sus procesos y finalidades. Algunos autores explican que ese hecho es producto indirecto de la ambigüedad con el que este campo se ha configurado, incluyendo las políticas educativas correspondientes (Cohen, Pouyaud, Drabik, Aisenson, Bernaut, Abdou, y Guichard, 2018; Grañeras y Parras, 2009). En el acervo conceptual de las diferentes profesiones que convergen en la práctica de la orientación escolar de Colombia no se ha logrado problematizar estructuralmente este campo. Ideas relativas a la intervención, pedagogía, contención, ayuda, y por supuesto, la escuela; están combinadas en las profesiones afines a la orientación pero con distinta ponderación en cada una. 
En Bogotá hay 1493 docentes orientadores en el sector oficial, distribuidos proporcionalmente según el tamaño de cada una de sus 20 localidades (SED, 2017). Una investigación sobre el rol y la función de la orientación escolar en la ciudad concluyó mostrando dos grupos de tensiones o problemas. Uno, de orden epistemológico, referido a la definición de la orientación escolar; y el otro, de orden metodológico respecto a su práctica cotidiana en los colegios (SED y Fundación para la Reconciliación, 2014). En otras palabras, ¿qué es la orientación escolar y cómo se hace? Describir los principales rasgos de la percepción social de la orientación escolar desde la perspectiva de los docentes orientadores es una forma para contribuir a su respuesta.

\section{Método}

Estudio descriptivo cuantitativo en la que participaron 1007 docentes orientadores de Bogotá; (90.4\%) del sector oficial y $(9.6 \%)$ del sector privado. Su formación de pregrado se presenta en la tabla 1.

\section{Tabla 1}

Formación de pregrado de los orientadores participantes

\begin{tabular}{lcc}
\hline Formación de pregrado & Frecuencia & Porcentaje \\
\hline Psicología & 510 & 46.1 \\
Licenciatura en Psicopedagogía & 307 & 27.7 \\
Trabajo social & 169 & 15.3 \\
Otro (Fonoaudiología, Terapia del lenguaje, & 121 & 10.9 \\
Sociología) & 1007 & 100 \\
Total & & \\
\hline
\end{tabular}

Instrumentos

Se diseñó una encuesta de 10 ítems sobre los aspectos más relevantes de la orientación escolar con opción de respuesta tipo Likert. Su validación se realizó a través de 3 jueces expertos en este campo. Los ítems 6, 7 y 9 se redactaron de modo negativo para mejorar su verosimilitud. La encuesta se presentó como parte del registro al Congreso de Orientación y Asesoría Escolar de agosto de 2018 en la página web de la Secretaría de Educación de Bogotá (tabla 2). Se atendieron los procedimientos legales sobre el manejo de datos personales y la confidencialidad de la información en investigación con humanos. 


\section{Tabla 2}

Lista de ítems de la encuesta diligenciada

\section{Afirmación}

1. El orientador es valorado positivamente en la escuela

2. El trabajo que realizo con padres de familia cumple mis expectativas

3. El orientador escolar $\mathrm{NO}$ es un maestro en sentido estricto

4. Tengo la formación necesaria para desempeñar mi trabajo como orientador

5. Las funciones de orientación escolar están sobrecargadas

6. Los estudiantes sienten que consultar al orientador es vergonzoso

7. Mi capacidad para transformar problemas en la escuela es mínima

8. Las políticas educativas sobre orientación son inconsistentes

9. Me siento desbordado emocionalmente con las situaciones que enfrento en mi trabajo como ori 10. Mi relación con los maestros es satisfactoria

\section{Procedimiento}

Una vez construida la base de datos se realizaron dos actividades: (a) análisis descriptivos de frecuencias para cada uno de los 10 ítems, y (b) tablas de contingencia entre la variable formación de pregrado y cada uno de los 10 ítems calculando el estadístico chi cuadrado (X2), para explorar alguna asociación significativa, con margen de error de 0.05 . Las variables fueron tratadas en su naturaleza nominal y ordinal según la especificidad de las respuestas de la escala Likert empleada. Para el análisis se usó el programa SPSS.

\section{Resultados}

En general los orientadores perciben favorablemente la orientación escolar. El promedio del porcentaje en las opciones "totalmente de acuerdo" y "de acuerdo" fue mayor que en el resto de las opciones de respuesta en todos los ítems positivos (68.7\%). Igualmente en los ítems negativos (6, 7 , 9 ) el promedio del porcentaje fue mayor en las opciones "totalmente en desacuerdo" y "en desacuerdo" que en el resto de las opciones (65.87\%). Esto indica una autocomprensión de rol del orientador en la escuela muy interesante, entre otras razones, por los múltiples inconvenientes que enfrentan en sus actividades cotidianas.

La descripción de frecuencias y porcentajes de respuesta para cada ítem muestra tres grupos de tendencias (tabla 3). En el primero se ubican los ítems con una tendencia alta en una de las direcciones de opción de respuesta $(4,5,7$ y 10$)$, y en el segundo, los ítems con una tendencia 
similar o más horizontal entre las opciones de respuesta (3 y 9). Los demás ítems se ubicaron en un punto medio entre las dos tendencias mencionadas.

Ítems con alta tendencia en una dirección de respuesta

La afirmación "tengo la formación necesaria para desempeñar mi trabajo como orientador" obtuvo $86.5 \%$ entre las opciones "de acuerdo" y "totalmente de acuerdo". Esto es llamativo dada la ausencia en el país de programas de formación específica en orientación escolar o educativa. Aunque en la encuesta no se discriminó el tiempo de experiencia profesional de los participantes pueden deducirse dos asuntos. De un lado que en los programas de formación de las profesiones afines existen contenidos curriculares respecto a la orientación, y de otro, que la formación de los orientadores se realiza "in situ". Los dos casos merecen revisiones más puntuales.

\section{Tabla 3}

Descripción de frecuencias y porcentaje para cada uno de los ítems

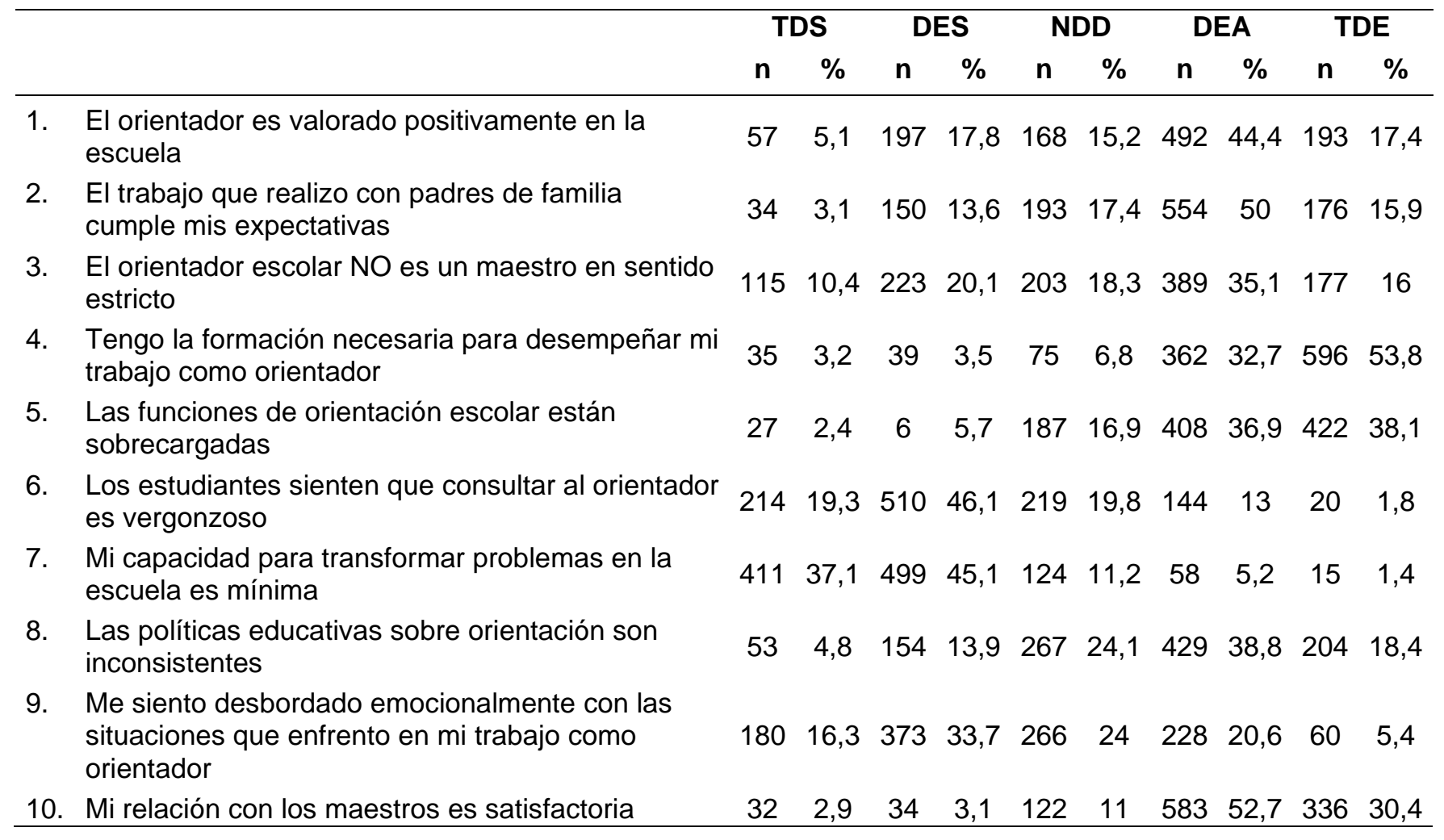

TDS=Totalmente en desacuerdo. DES=En Desacuerdo. NDD=Ni en acuerdo ni en desacuerdo. DEA=De acuerdo. TDE= Totalmente de acuerdo.

El ítem "mi relación con los maestros es satisfactoria" obtuvo 83.1\% entre respuesta "de acuerdo" y "totalmente de acuerdo". Varios autores han argumentado que la orientación no se circunscribe a un proceso individual, solamente referido al orientador (Grañeras y Parras, 2009). La colaboración de los 
maestros en los procesos correspondientes está ligada a la representación del lugar de los aspectos personales y emocionales en el aprendizaje. Es conveniente fortalecer la relación entre los orientadores y los maestros en la escuela porque, al final, favorece a toda la comunidad escolar. En ese sentido, valdría la pena examinar la percepción de esa relación pero desde la perspectiva de los maestros.

La afirmación "mi capacidad para transformar problemas de la escuela es mínima" presentó $82.2 \%$ entre las opciones "totalmente en desacuerdo" y "en desacuerdo". La cotidianidad profesional del orientador es complicada porque siempre hay asuntos urgentes para atender. Si bien percibir la propia capacidad de transformación no implica la transformación por sí, es esencial que los orientadores se perciban a sí mismos como tales. De lo contrario los motivos para desempeñar el trabajo se diluirían paulatinamente. Es fundamental desarrollar programas que mantengan y cualifiquen esa capacidad de transformación.

El ítem "las funciones de la orientación escolar están sobrecargadas" obtuvo entre las opciones "de acuerdo" y "totalmente de acuerdo" $75 \%$. Este resultado no es sorprendente ya que la tasa de estudiante por orientador en Bogotá es de 521 (MEN, 2017). En otras entidades territoriales del país el promedio es mucho mayor. A esto se suma la ambigüedad de las actividades que les son asignadas. Este es un asunto que merece atención especial porque afecta tanto la efectividad de las acciones realizadas por los orientadores como su propia salud física y emocional.

\section{Ítems con tendencia similar entre las opciones de respuesta}

A propósito de ese último asunto, la afirmación "me siento desbordado emocionalmente con las situaciones que enfrento en mi trabajo como orientador" obtuvo un porcentaje más uniforme entre las opciones de respuesta comparado con los demás ítems: "totalmente en desacuerdo" (16.3\%), "en desacuerdo" (33.7\%), "ni de acuerdo ni en desacuerdo" (24\%), "de acuerdo" (20.6\%) y "totalmente de acuerdo" (5.4\%). Es probable que los orientadores aprendan a manejar sus emociones durante su experiencia profesional o que recurran a estrategias que les permitan hacerlo. En usual que ellos afronten situaciones altamente estresantes. Uno de cada cuatro de los orientadores que diligenciaron la encuesta se siente desbordado emocionalmente. Esta es una cifra preocupante.

El ítem "el orientador escolar $\mathrm{NO}$ es un maestro en sentido estricto" obtuvo las siguientes respuestas: "totalmente en desacuerdo" (10.4\%), "en desacuerdo" (20.1\%), "ni de acuerdo ni en desacuerdo" (18.3\%), "de acuerdo" (35.1\%) y "totalmente de acuerdo" (16\%). Se trata del conjunto de porcentajes más equilibrado en las opciones, de todas las afirmaciones. Esto muestra una discusión dentro del gremio de los orientadores escolares respecto a esa denominación, derivada de las políticas educativas correspondientes. Los interrogantes derivados se centran en la figura del orientador como parte de la cultura escolar.

\section{Ítems con tendencia de respuesta intermedia}

Las cuatro afirmaciones restantes obtuvieron porcentajes de respuesta favorable pero moderada, no tan alta como en los ítems 4, 5, 7 y 10, ni tan similar como en los ítems 3 y 9 . Estas son "el orientador es valorado positivamente en la escuela", "el trabajo que realizo con los padres de familia cumple mis 
expectativas", "los estudiantes sienten que consultar al orientador es vergonzoso" y "las políticas educativas sobre la orientación son inconsistentes". Esto significa que en estos casos existe consenso pero con una disidencia importante.

En el cálculo del chi cuadrado (X2) no se identificó claramente si esa disidencia pertenece a un grupo de orientadores discriminada según su formación de pregrado. Como la encuesta no recopiló información sobre género, edad, tiempo de experiencia o formación de posgrado, tampoco se lograron establecer clústeres. Por lo tanto las ideas relativas a estos cuatro ítems podrían ser usadas para investigaciones puntuales, incluso, de orden cualitativo.

Por qué un orientador no se sentiría valorado positivamente en la escuela, o por qué no se cumplirían las expectativas del trabajo con padres de familia o quienes creerán que consultar al orientador en vergonzoso y cuáles orientadores piensan que las políticas educativas afines son consistentes. Todos estos interrogantes abren posibilidades de estudios para avanzar en la comprensión de la orientación escolar en Bogotá.

\section{Formación pregradual del orientador y respuestas para cada ítem}

La variable que se pretendía contrastar con las respuestas para cada ítem era la formación de pregrado del orientador. Las proporciones de los participantes fueron: psicología (46.1\%), licenciatura en psicopedagogía (27.7\%), trabajo social (15.3\%) y otros, fonoaudiología, terapia del lenguaje, sociología (10.9\%). El cálculo de chi cuadrado (X2) mostró una asociación con el ítem "El orientador escolar NO es un maestro en sentido estricto", 32.152, con un margen de error de 0.05 (tabla 4).

Tabla 4

Cálculo de chi cuadrado (X2) entre formación de pregrado y cada ítem

\begin{tabular}{lc}
\hline Ítem & Pregrado X2 \\
\hline 1. El orientador es valorado positivamente en la escuela & 10,854 \\
2. El trabajo que realizo con padres de familia cumple mis expectativas & 20,452 \\
3. El orientador escolar NO es un maestro en sentido estricto & 32,152 \\
4. Tengo la formación necesaria para desempeñar mi trabajo como orientador & 13,131 \\
5. Las funciones de orientación escolar están sobrecargadas & 17,435 \\
6. Los estudiantes sienten que consultar al orientador es vergonzoso & 19,651 \\
7. Mi capacidad para transformar problemas en la escuela es mínima & 10,551 \\
8. Las políticas educativas sobre orientación son inconsistentes & 16,521 \\
9. Me siento desbordado emocionalmente con las situaciones que enfrento en mi & \\
trabajo & 15,287 \\
10. Mi relación con los maestros es satisfactoria & 12,238 \\
\hline
\end{tabular}

12 gl. X2 crítico=21.03, $p<0.05$

El hecho de que en 9 de los 10 ítems no exista asociación es un resultado significativo. Implica que la formación de pregrado no influye en el campo perceptual de las ideas implícitas en cada uno 
de ellos. Por lo tanto es razonable argumentar que el orientador escolar realmente se forma "in situ", con todas las ventajas y las limitaciones que eso conlleva. De ese modo la actividad del orientador adquiere relevancia como espacio de formación profesional. Los detalles de ese proceso deben estudiarse para proponer programas de cualificación relevantes.

Asimismo la influencia de la formación de pregrado del orientador en su auto representación como maestro es muy interesante. La ponderación de los porcentajes muestra que los que menos se ven a sí mismos como maestros son los psicólogos y los que más se ven a sí mismos como maestros son los licenciados en psicopedagogía (tabla 5). Esto tiene sentido dada la especificidad pedagógica de este último grupo de profesionales. Valdría la pena profundizar en las prácticas específicas de orientación para cada uno de estos dos grupos.

\section{Tabla 5}

Tabla de contingencia de porcentajes formación de pregrado / ítem 3

\begin{tabular}{lccccc}
\hline & \multicolumn{5}{c}{ El orientador NO es un maestro en sentido estricto } \\
Pregrado/Opción & TDS & DES & NDD & DEA & TDE \\
\hline Psicología & 7,1 & 18 & 16,7 & 37,6 & 20,6 \\
Otro & 10,7 & 19,8 & 19 & 37,2 & 13,2 \\
Trabajo social & 11,8 & 20,7 & 21,9 & 33,1 & 12,4 \\
Licenciado en Psicopedagogía & 15 & 23,5 & 18,9 & 31,3 & 11,4 \\
\hline
\end{tabular}

TDS=Totalmente en desacuerdo. DES=En Desacuerdo. NDD=Ni en acuerdo ni en desacuerdo. DEA=De acuerdo. TDE= Totalmente de acuerdo; $n=1007$.

Esto asienta un problema de investigación sustancial. Puede denominarse la dimensión pedagógica de la orientación entendida como la problematización de ese fenómeno dentro de la cultura escolar. Para ello tendría que fortalecerse tanto el acervo conceptual de la orientación como la reflexión sistemática de su práctica. Desde luego, una concepción epistemológica también debería estar presente. Las implicaciones de la auto representación de los orientadores como maestros se centran en la actividad concreta de su trabajo y en los motivos que los sustentan.

\section{Discusión y Conclusiones}

Este estudio tuvo el propósito de describir la percepción social hacia la orientación escolar en 1007 orientadores de Bogotá. Para ello se preguntó sobre el grado de acuerdo en 10 ítems que contenían las ideas más significativas en torno al ejercicio de la orientación en los colegios de la ciudad. La única variable que se registró para realizar comparaciones fue la formación de pregrado del orientador, ya que en Colombia no existe ningún pregrado específico en esta área. En este trabajo hay un interés por la formación de los orientadores escolares del país. 
Un aspecto importante de esta encuesta es la representatividad de la muestra de participantes. 1007 orientadores de 1493 existentes, en el sector oficial, constituye el 67.4\%. El Congreso de Orientación y Asesoría Escolar organizado por la Secretaría de Educación del Distrito de Bogotá fue una ocasión inmejorable. A propósito este es uno de los eventos que impulsa esa entidad en conjunto con los gremios de orientadores de la ciudad. Una práctica que debe mantenerse y cualificarse.

Los resultados generales de la encuesta coinciden con el panorama de la investigación afín en Latinoamérica. En la mayoría de los casos existe favorabilidad hacia la orientación escolar, manifestada en su relevancia y en el reconocimiento de su incidencia en los procesos educativos (Delgado, 2016; García, 2015; López y Manzano, 2019; Muñoz, 2016; Nascimiento, Querino, Maciel y Da Cunha, 1983). En estos estudios se subraya la influencia del bienestar social y emocional de los estudiantes en su aprendizaje. Además del maestro, se asigna al orientador escolar la responsabilidad de atender este fenómeno.

La necesidad de un orientador en la escuela está sustentada en esa creencia. Debe existir un profesional dedicado a atender los problemas sociales y afectivos de la comunidad escolar. Al fin y al cabo la mayoría de los asuntos en los departamentos de orientación son de ese tipo: ideaciones suicidas, agresión, autoestima, sexualidad, etc. Todo aquello que pueda apoyar a los estudiantes para tener éxito académico será bienvenido en la escuela; la cuestión es determinar la pertinencia y los recursos empleados en ese tipo de apoyo.

La escuela es una institución que se está transformando hacia un lugar de contención emocional. Esto se refleja en las valoraciones que otros miembros de la comunidad escolar, incluyendo los maestros de aula, realizan sobre la actividad de los orientadores escolares. Por ejemplo, Güler y Capri (2019), estudiaron las opiniones de maestros respecto a los procesos de consejería en escuelas de Turquía y encontraron una alta favorabilidad, fundamentada en el conocimiento de los maestros en sus actividades. Todo indica que cada vez se otorga más importancia al apoyo de procesos no cognitivos relativos al aprendizaje y la enseñanza.

Según los resultados, la conciencia que tienen los orientadores de su rol dentro de ese apoyo es clara. Las altas tendencias de favorabilidad en las afirmaciones "tengo la formación necesaria para desempeñar mi trabajo como orientador", y "mi relación con los maestros es satisfactoria", junto con la alta tendencia de desacuerdo en la afirmación "mi capacidad para transformar problemas en la escuela es mínima"; es una muestra de ello. En cierto sentido los orientadores creen firmemente en la relevancia de su trabajo dentro de la escuela.

Esa firmeza también se ve probada por las dificultades cotidianas que enfrentan en su trabajo. Incluso, la afirmación "las funciones de la orientación escolar están sobrecargadas" obtuvo una alta tendencia de acuerdo. Al respecto puede sugerirse que la actividad de la orientación en la escuela suscita en los orientadores una capacidad de resistencia especial a las dificultades. Es probable que la intensidad emocional de su actividad les obligue a desarrollar estrategias que mejoren sus reacciones hacia la frustración.

A propósito, los resultados menos dispares en la afirmación "me siento desbordado emocionalmente con las situaciones que enfrento en mi trabajo como orientador" pueden interpretarse en esa línea, con una presunción: los orientadores con mayor experiencia han desarrollado más estrategias para manejar adecuadamente sus emociones en su trabajo que los orientadores con menos experiencia. Debe recordarse que no se encontró asociación entre este ítem y la formación de pregrado de los participantes. La experiencia de los orientadores emerge como un campo de estudio significativo.

Respecto a los resultados de las afirmaciones "el orientador es valorado positivamente en la escuela", "el trabajo que realizo con los padres de familia cumple mis expectativas", "los estudiantes 
sienten que consultar al orientador es vergonzoso" y "las políticas educativas sobre la orientación son inconsistentes", que presentaron favorabilidad moderada con la presencia de un subgrupo disidente, puede afirmarse que estos constituyen focos para entender rasgos mucho más particulares de este fenómeno.

En otras palabras, dichos resultados pueden condensar la ambigüedad de las funciones y representaciones de los demás miembros de las comunidades educativas respecto a la orientación escolar. En los estudios afines siempre existe esta preocupación, traducida en la heterogeneidad de expectativas sobre el propósito y alcance de su trabajo entre los demás miembros de las comunidades escolares (Amer y Mir, 2018; Campo y Labarca, 2009; Cano, Mayoral, Liesa y Castellano, 2013; Castillo y Torres, 2013; Hincapié y Arbeláez, 1979, Peña, 2006; Rodríguez, 2014).

¿Qué factores intervienen en la valoración de un orientador escolar por parte de su comunidad educativa?, ¿cómo lograr satisfacción en el trabajo con padres de familia?, ¿los estudiantes que se avergüenzan de consultar al orientador tienen características similares?, y ¿cuál es el grado de conocimiento de los orientadores sobre las políticas educativas en orientación? Este es un conjunto de cuestiones que pueden fundamentar investigaciones más focalizadas.

Algunos estudios muestran que factores específicos del profesorado, como su edad, su género, o el contenido de su enseñanza, no están estadísticamente relacionadas con sus creencias respecto a los programas de orientación escolar (Aluede y Egbochuku, 2007). Incluso, se está promoviendo la enseñanza de habilidades propias de la orientación al resto de maestros de aula, bajo la perspectiva de intervenciones integradas (Batini, Barolicci, y De Carlo, 2019; Pharis, Wu, Sullivan, y Moore, 2019). Esto plantea una relación entre orientadores y maestros que podría estudiarse con más profundidad.

En todo caso estas ideas revelan parte de la heterogeneidad con la cual se ha configurado la orientación escolar en Colombia. Además se suma la singularidad, que paradójicamente, es un aspecto común del éxito de intervenciones o programas escolares (Baquero, Terigi, Toscano, Brisciol, y Sburlatti, 2009; Beyebach y De la Vega, 2018). Lo que funciona en una escuela no necesariamente funciona en otra, y lo que explica el funcionamiento de un programa es, precisamente, los aspectos particulares de una institución educativa determinada. Así las cosas se plantea un panorama amplio pero con rutas específicas para estudiar la orientación escolar en Bogotá.

La asociación entre la formación de pregrado del orientador y su auto representación como maestro es, en parte, lógica. Indudablemente un licenciado en psicopedagogía tiene una formación pedagógica más sólida que un psicólogo o un trabajador social. Esto en distinta medida afectará los motivos y las actividades de su trabajo. Tal parece que la incorporación de la orientación a la escuela es un proceso que está inacabado, que está en marcha (Menenes, 2001). Aún persiste su impronta vocacional, y por influencia de la psicología, también existen reminiscencias de su origen en el modelo médico.

Esto origina una discusión muy importante. Se trata de pensar en la orientación escolar como una actividad docente. ¿El orientador escolar enseña o forma algo?, si es así ¿qué es y cómo lo hace? La incidencia de las prácticas de orientación sobre los estudiantes puede entenderse como una formación de sus virtudes, emociones, y formas de ver el mundo y de relacionarse con uno mismo (Romito, 2019). También existen iniciativas para estimular la participación de los orientadores en diseños curriculares que promuevan la prevención de problemas emocionales (Dack y Merlin, 2019).

La problematización y la lectura pedagógica de la orientación pueden comenzarse con esa cuestión: distinguir entre un orientador que se ve a sí mismo como maestro y uno que no lo hace, junto con las ideas subyacentes. También es fundamental articular conceptualmente la orientación 
con las teorías sobre la escuela, y más allá, con su sociología. En todo caso, la escuela es una institución que en la medida de sus posibilidades atiende las dificultades sociales circundantes.

Por consiguiente, los problemas epistemológicos continúan vigentes y adquieren supremacía sobre los problemas metodológicos, según el reporte de la Secretaría de Educación y la Fundación para la Reconciliación (2014). No solo se trata de encontrar formas eficaces de resolver problemas, lo cual es necesario, sino también de pensar por qué y para qué hacerlo. La instrumentalización de la orientación escolar provocaría una desconexión de los problemas socioeducativos del país.

Si como indica la teoría, la percepción social de un fenómeno está estrechamente ligada a la experiencia, y los resultados de esta encuesta muestran mayor consenso que disenso, y además, solo en uno de los 10 ítems se encontró asociación con la formación pregradual; entonces la actividad in situ de los orientadores adquiere relevancia intelectual y práctica. Ya se mostraron algunas ideas de investigaciones puntuales, que pueden desarrollarse en otras ciudades de Colombia, con métodos cuantitativos y cualitativos. La reflexión sistemática sobre la práctica continúa siendo una fuente de conocimiento de la actividad pedagógica en la escuela.

Finalmente, quedan planteadas algunas cuestiones sobre la práctica de los orientadores escolares. Estudios posteriores podrían centrarse en la incidencia de otras variables como género, edad, o años de experiencia en la percepción social de esa actividad, usando escalas con más ítems (Morais, Condon, Blanco y Suárez, 2019). También sería interesante explorar dicha percepción en otros miembros de las comunidades educativas: estudiantes, maestros y padres de familia. Esto aportaría información para evaluar el trabajo de los orientadores y parte de su impacto en los procesos formativos de la escuela.

\section{Referencias bibliográficas}

Aluede, O. y Egbochuku, E. (2007). he Influence of Personal Characteristics on Secondary School Teachers' Beliefs about School Guidance and Counselling Programs. Education, 127(3), 440-446.

Amadeo, H. (2007). La orientación educativa actual en la educación secundaria obligatoria de España. Pedagogía y Saberes. 27(1), 27-31.

Amer Fernández, J., y Mir Gual, A. (2018). Los procesos de implementación de los programas de mejora del aprendizaje y el rendimiento (PMAR). Perspectivas del profesorado, el alumnado y las familias. Revista Española de Orientación y Psicopedagogía, 28(3), 133-150.

Baquero, R., Terigi, F., Toscano., A., Brisciol, B. y Sburlatti, S. (2009). Variaciones del régimen académico con población vulnerable. Un estudio de casos en el área metropolitana de Buenos Aires. Revista Iberoamericana sobre Calidad, Eficacia y Cambio en Educación, 7(4) 293-319.

Batini, F., Barolicci, M. y De Carlo, E. (2019). I Feel Good at School! Reducing School Discomfort Levels through Integrated Interventions. Athens Journal of Education, 6(3), 209-221.

Bejarano, M. y Huertas, D. (2017). La orientación educativa en Colombia. Bogotá: Uniediciones. 
Beyebach, M. y De la Vega, M. (2018). Intervención escolar centrada en soluciones: conversaciones para el cambio en la escuela: un manual práctico para profesionales de la educación. Barcelona: Herder.

Campo, M. y Labarca. C. (2009). Representaciones sociales del rol orientador del docente en estudiantes de educación. Revista de Ciencias Sociales.15 (1), 160-174.

Cano, M., Mayoral, P., Liesa. E. y Castellano, M. (2013). Valoración de las funciones del profesor de orientación educativa en Cataluña. Revista Española de Orientación y Psicopedagogía, 23(3), 8097.

Castillo, M. y Torres, J. (2013). Percepción del orientador escolar por la comunidad educativa. International Journal of Developmental and Educational Psychology. 25 (1), 469-476.

Cohen-Scali, V. et al. (Eds.) (2018). Interventions in Career Design and Education. New York: Springer.

Dack, H., y Merlin, C. (2019). Improving Classroom Guidance Curriculum with "Understanding by Design". Professional Counselor, 9(2), 80-99.

Delgado, A. (2016). Orientación escolar en el marco de la convivencia escolar, una mirada desde las representaciones sociales: El caso de la institución educativa departamental Alfonso López Pumarejo del municipio de Nemocón. (Tesis de maestría). Universidad Pedagógica Nacional. Bogotá, Colombia.

García, J. (2015). Hacia el reconocimiento del papel del orientador escolar en gestión educativa estudio de caso colegio Jaime Quijano Caballero. (Tesis de pregrado). Universidad Pedagógica Nacional. Bogotá, Colombia.

Grañeras M., y Parras, A. (2009). Orientación educativa: Fundamentos teóricos, modelos institucionales y nuevas perspectivas. Madrid: Ministerio de Educación.

Güler, M., y Capri, B. (2019). Teachers' opinions on the pre-school counselling program. Pegem Journal of Education and Instruction, 9(2), 521-546.

Hernández, O. (2016). La exacerbación de los saberes psicológicos en contextos escolares. En M. Riaño, J. Torrado, S. Carrillo, M. Bautista, y E. Díaz (Comps.) Contexto educativo: convergencias y retos de la psicología educativa. Cúcuta: Ediciones Universidad Simón Bolívar.

Hincapié, C, y Arbeláez, M (1979). Actitudes de la comunidad educativa de los colegios nacionales de Bogotá hacia los servicios de orientación y asesoría escolar. (Tesis de maestría). Universidad Pedagógica Nacional. Bogotá, Colombia.

López Díez-Caballero, M., y Manzano-Soto, N. (2019). Valoración del nuevo modelo de orientación implementado en la comunidad autónoma de Cantabria por parte de los orientadores educativos. REOP - Revista Española de Orientación y Psicopedagogía, 30(2), 108-127.

Martínez, M. (2002). La orientación escolar en el marco de la orientación colombiana. Primer congreso de la orientación en países bolivarianos. Caracas: Unicentral.

Ministerio de Educación Nacional (Comunicación personal del 30 de octubre de 2017) mencionan los datos de los orientadores escolares nombrados en cada entidad territorial junto con la matricula oficial de cada uno. Recuperado de https://www.mineducacion.gov.co/portal/ 
Morais, M., Condon, J., Blanco, J., y Suárez, E. (2019). Inclusion, diversity and equity: Design and validation of on opinion questionnarie adressed to school guidance counsellors. Revista de Investigación Educativa, 37(2), 505-52

Muñoz, B. (2016). Hojas Sueltas. En Revista Mexicana de Orientación Educativ, 13(30), 54-58.

Myers, D. (2000). Psicología social. México: McGraw-Hill.

Nascimento, M., Querino, A., Maciel, A., y Da Cunha, A. (1983). Percepções de estudantes acerca da orientação recebida da universidade - um estudo interinstitucional (UFAM-UNIR e UFGD). Revista Amazónica, 3(2), 18-31.

Nater, C, y Zell, E. (2015). Accuracy of Social Perception: An Integration and Review of MetaAnalyses. Social and Personality Psychology Compass, 9(9), 481-494.

Pharis, T., Wu, E., Sullivan, S., y Moore, L. (2019). Improving Teacher Quality: Professional Development Implications from Teacher Professional Growth and Effectiveness System Implementation in Rural Kentucky High Schools. Educational research quarterly, 42(3), 29-48.

Peña, F. (2006). Identidad profesional: La problemática de la psicopedagogía. Pedagogía y Saberes, 25(1), 109-120.

Rodríguez, H. (2014). Reflexiones acerca de la identidad profesional del docente orientador en colegios distritales de la localidad de Bosa (tesis de maestría) Universidad Pedagógica Nacional, Bogotá, Colombia.

Romito, M. (2019). Governing through Guidance: An Analysis of Educational Guidance Practices in An Italian Lower Secondary School. Discourse: Studies in the Cultural Politics of Education, 40(6), 773-788.

Secretaría de Educación de Bogotá (Comunicación personal del 9 de noviembre de 2017) menciona los datos de docentes orientadores en cada una de las 20 localidades de la ciudad.

Secretaría de Educación de Bogotá y Fundación para la reconciliación (2014). La convivencia escolar, cuestión humana: Reflexión acerca del rol y función de la orientación escolar en asuntos de convivencia. Bogotá: Alcaldía Mayor de Bogotá.

Fecha de entrada: 28 noviembre 2018

Fecha de revisión: 16 diciembre 2019

Fecha de aceptación: 03 febrero 2020 